\title{
A low quiescent power wireless rotating machinery condition monitoring system
}

\author{
Yan Chen, Zaffir Chaudhry, Joseph Mantese \\ Raytheon Technologies Research Center, East Hartford, Connecticut, 06108, USA \\ yan.chen1@rtx.com \\ zaffir.chaudhry@rtx.com \\ joseph.mantese@rtx.com
}

\begin{abstract}
Vibration-based monitoring of rotating machinery is rapidly evolving within the aerospace industry with priority on anticipating and detecting impending failures. The workhorse of such prognosticating system remains the piezoelectricbased accelerometer which requires a wired-harnesses, connectors, significant power, signal conditioning, etc. To address the challenge of a complex wired sensing system entailing the use of multi-sensing nodes, Raytheon Technologies Research Center (RTRC) along with Collins Aerospace and Sandia National Laboratories (SNL) have jointly developed an Aluminum-Nitride Resonant Integrated Accelerometer Sensors (ARISE).

ARISE is a low power alternative to a conventional wired vibration-based monitoring system. This self-contained sensor system includes: (1) a low quiescent power sensing element with a wake-up module, (2) a wireless communication module that draws power only when triggered by physical trigger, characteristic of the fault signatures, and (3) a low capacity energy source as provided by a coin-cell battery. Leveraging work performed under Defense Advanced Research Projects Agency (DARPA) NZero program, this wireless health monitoring system can operate in a quiescent low power mode $(\sim 10 \mathrm{nW})$ for a period of several years without servicing. With an exceedance above a preset vibration level (at characteristic frequencies of the system under test), the sensor wakes up and wirelessly sends a warning of a precursor-to-failure.
\end{abstract}

The ARISE sensor and wake-up module package has been validated with a replicated vibration signature acquired from a select rotating machinery subject to progressive damage at the Structural Dynamics Laboratory at Raytheon Technologies Research Center RTRC. The failure precursor

\footnotetext{
Yan Chen et al. This is an open-access article distributed under the terms of the Creative Commons Attribution 3.0 United States License, which permits unrestricted use, distribution, and reproduction in any medium, provided the original author and source are credited.
}

is successfully detected by the sensor which activates the wake-up module.

This research was conducted with funding from the DARPA Micro Technology Office (MTO), under ARISE program.

\section{BACKGROUND}

Prognostics and Health Monitoring (PHM) has rapidly evolved over the last decade with both industry and the DoD coming to the realization that the true value proposition of system health monitoring reaches significantly beyond fault identification, into Condition-based Maintenance (CBM). Indeed, as outlined in the DoD Condition Based Maintenance Plus Guidebook, CBM is currently viewed by the DoD as the most economic means of high asset readiness. CBM can be broken down into two elements; the inspection and replacement of life-limited parts in expensive and limitavailability assets. Replacing parts under Schedule Based Maintenance (SBM) in limited asset systems adds significantly to the cost of operations, reduces combat readiness as a result of the unnecessary removal of an asset, and requires significant capital expenditure to have multiple platforms present on a military installation to provide minimum level combat readiness. Health and Utilization Monitoring Systems (HUMS) primarily use high power consumption Commercial-Off-The-Shelf (COTS) sensors to replace manual inspections, they come with the price of added weight, cost, complexity, and unreliability due to the increased interconnects and power needs of the sensing elements. One of the central elements of CBM, in particular, is the vibration-based rotating machinery monitoring. Consequently, while there has been much research and many advancements related to improved vibration signalprocessing algorithms, the state-of-art in sensors has remained essentially unchanged for the last two decades. HUMS continues to use piezoelectric-based conventional accelerometers which require wire harnesses, connectors, significant power, signal conditioning, etc. Indeed, in many instances the entire HUMS monitoring system's (including sensors) own Mean Time Between Failure (MTBF) is less 
than the mechanical system being monitored. The N-Zero based proposed sensor systems developed by Sandia National Laboratories, as described in a series of papers from Griffin, McClanahan, Clews, Reger, Yen et al (2017), even though not an exact replacement for a wired accelerometer, represents a paradigm shift in rotating machinery monitoring and will provide the critical component for a low-cost practical and implementation of CBM systems for essentially all rotating machines.

The principal failure mode of rotating machines is the spallation of bearing race under high loads when operated at high speed and temperature, see Figure 1 as an example of bearing race spallation. This failure mode is due to the "pitting" of the "race" of the bearing, leading to a "spall" that can grow quickly, resulting in catastrophic failure. In the section below, we describe the different phases of bearing degradation and detectability and further our approach of using unique system resonant frequencies to achieve the earliest signs of bearing degradation. Ironically, however, aerospace systems are migrating to even higher rotating speeds, temperatures, and contact stresses to reduce overall system size. The most successful methods of diagnosing rotating equipment and bearing failure relies upon attaching single or multi-axis accelerometers to the equipment and monitoring the vibration-signals to detect progressive system degradation and failure. These techniques are well developed, highly successful, and are an integral part of deployed HUMS; but are often implemented only in "must have" applications; primarily because such devices must be: (1) electrically wired to provide the requisite power to the device. (2) Requires additional leads for communications. (3) Has a relatively large footprint (connectors) even where space is a premium. Consequently, a non-wired, low power accelerometer that is maintenance free for $\sim 5$ years and keyed to distinct failure modes of the rotating machinery enables a very attractive alternate and indeed a paradigm shift.
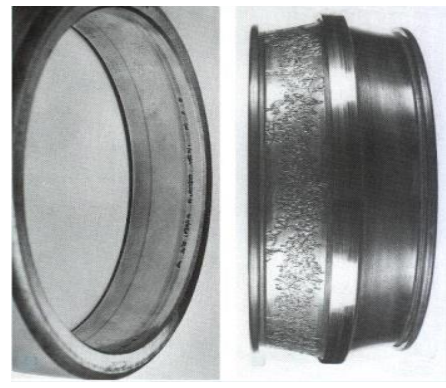

Figure 1. Bearing spallation

In industry and academia, the bearing degradation can be categorized by four stages, illustrated in Figure 2.

Stage 1: Micro defects are initiated, which excite energy in the very-high frequency range - Zone 4 in Figure 2. This minor damage is extremely hard to detect in the field environment and also is not of much interest because such nano-meter scale damage does not compromise bearing life in any immediate way. In fact such features can sometimes self-heal and thus are not subject to servicing or replacement.

Stage 2: Small defects, i.e. macro pitting, is developed which leads to repeated impacts between the ball and the race. The repeated impulse forces produce easily measurable changes at unique frequency/s - so called "carrier" frequency/s. The carrier frequency is an inherent resonance frequency of the structure, which is independent of speed and load condition, and thereby represents a key diagnostic frequency of the system that we intend to exploit with ARISE. The peak of impulse response at the select carrier frequency is proportional to the impact force or the damage severity. Figure 3 shows the energy evolution of a bearing degradation from Stage 1 to Stage 3. The speed independent 'carrier' frequencies are clearly visible as well as the growing amplitude at these frequencies.

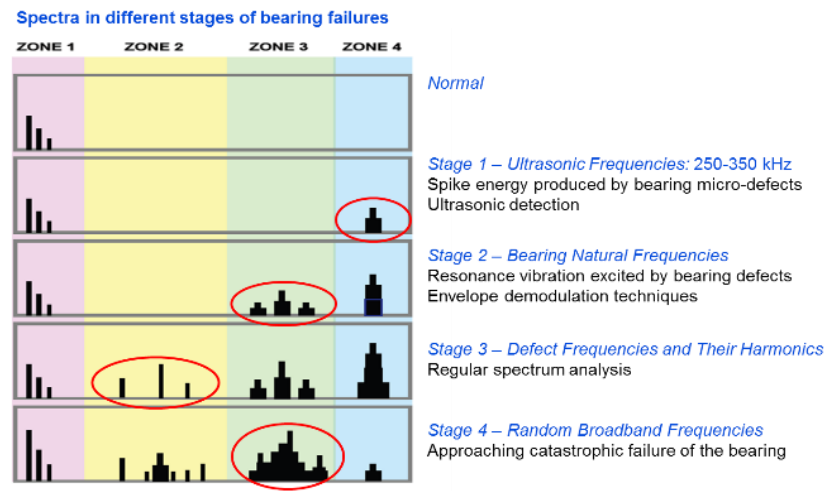

Figure 2. Bearing failure progression, symptom and detection

Waterfall plot of a bearing damage

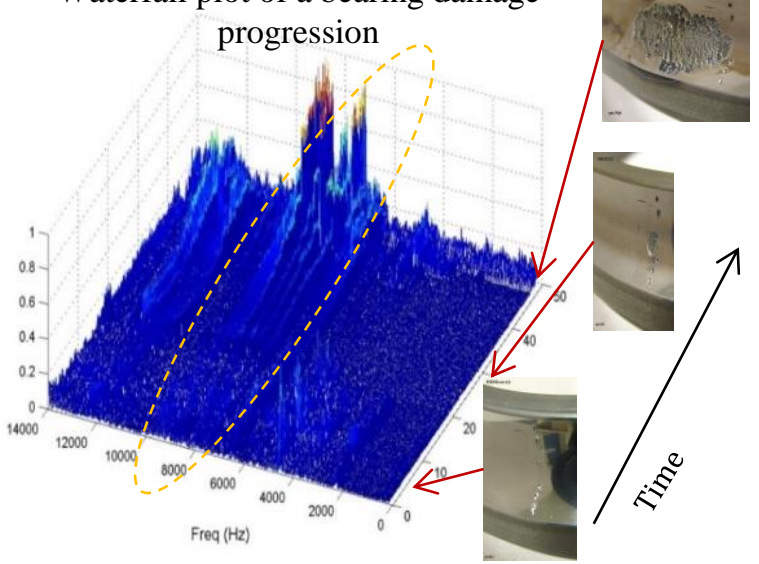

Figure 3. Example of a vibratory energy and damage evolution from Stage 1 to Stage 3. Very visible are the distinct "carrier" frequencies and the increasing amplitude with damage progression 
The industry is currently heavily investing in advanced algorithms targeting Stage 2 bearing degradation. Envelope demodulation methods, shown in Figure 4, are one the most advanced algorithms widely adapted by industry. The key element of this algorithm is the band-pass filter around the carrier frequency. The N-Zero based sensor technology is inherently a "band-pass filter", which can be readily implemented once the "carrier" frequency is identified. RTRC has established and demonstrated a method to identify the carrier frequency, which is presented by Chen, Chaudhry (2014).

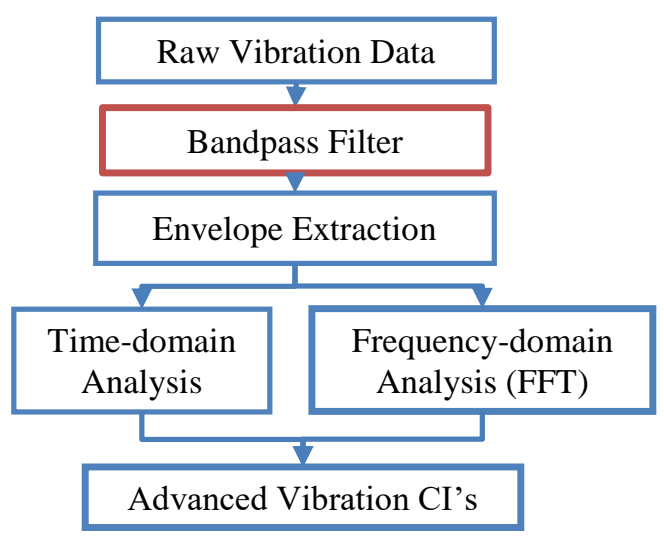

Figure 4. Block diagram of an advanced envelope demodulation algorithm

Stage 3: At this stage, the damage is fully developed and the "bearing fault frequencies" are visible and the safety of high value critical systems is not guaranteed. The "bearing fault frequencies", such as "inner race ball passage frequency" are related to the shaft rotation speed. Usually the classical methods target vibration magnitude at these frequencies. However, during this stage, the damage can grow fast and the remaining life can be very limited. For the rotary machine operating at a constant speed, those bearing fault frequencies are fixed. Therefore N-Zero sensors can be also designed at those frequencies, which will provide the industry an alternative low cost and practical bearing monitoring solution.

Stage 4: In this stage, the bearing damage is widespread with imminent system failure and there is no interest in detection this late as the entire asset is at risk of loss.

In summary, vibration-based bearing health monitoring methods target Stage 2 and Stage 3 damage. During the past decade, early indication of Stage 2 damage has received the most attention, and is also the focus of this effort. By targeting Stage 2, we believe that the proposed sensor will be a fundamental enabler of $\mathrm{CBM}$ for high value rotating engines and machines.

In the following sections of the paper, the vibration characteristics of the targeted application are discussed first. This is followed by the validation of the MEMS resonator sensor's performance, functionality of the combined sensor wakeup ASIC package, and finally a description and test results of the integrated Sensor-ASIC package subject to a replica of the actual vibration signature.

\section{ARISE UNDERLYING TECHNOLOGY}

The underlying sensor consists of a MEMS AluminumNitride resonant accelerometer tuned to a specific frequency, a wakeup ASIC and a wireless module. The schematic of the system is shown in Figure 5.

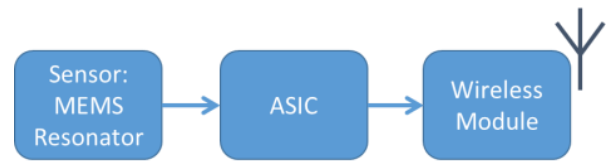

Figure 5. Schematic of the ARISE technology

The ARISE MEMS sensor is a mass-spring mechanical system, as illustrated in Figure 6, which is fabricated with a designated resonance frequency. For monitoring, the sensor is attached to the test article and subject to base excitation. When the excitation frequency coincides with the resonance frequency of the sensor, the sensor resonates and the spring deflection is maximized. The AIN film on the spring generates electrical charge when the spring deflects. A graphic summarizing the relevant die-level characteristics of the SNL sensor, which this program builds upon, is shown in Figure 6. A few key features to note are: The size of the MEMS sensor, and the match between experimental and model results. Table 1 below shows the high output despite the $5-20 \mathrm{~Hz}$ bandwidth making this very attractive for such PHM sensor applications.

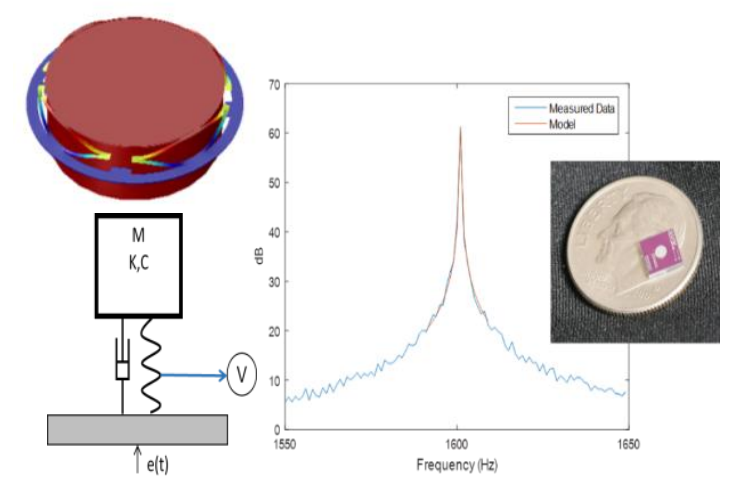

Figure 6. A Sandia N-Zero die-level measurements showing the ability to design in frequency and achieve very high sensitivity. The size of the MEMS sensor is also obvious.

The sensor output is fed into a wake-up ASIC. Once the sensor output voltage exceeds the preset threshold of the ASIC, the ASIC is tripped and energizes the wireless module through the coin cell battery which sends out a Fault Alert Signal. 


\begin{tabular}{|l|r|r|r|}
\hline & Quality Factor & Sensitivity & $\begin{array}{r}\text { Expected output } \\
\text { signal for 0.01-0.1 g }\end{array}$ \\
\hline \begin{tabular}{l|r|r} 
Quasi-static \\
Narrowband sensitivity
\end{tabular} & NA & $8.4 \mathrm{mV} / \mathrm{g}$ & $\mathrm{NA}$ \\
\hline $\begin{array}{l}\text { Moderate bandwidth } \\
(\sim 5 \mathrm{~Hz}) \text { sensitivity }\end{array}$ & 11,000 & $94,000 \mathrm{mV} / \mathrm{g}$ & $940-9,400 \mathrm{mV}$ \\
\hline
\end{tabular}

Table 1. Sensitivity of N-Zero sensor

The resonator sensor, in itself a passive device, which generates signal via a non-steady state acceleration of a MEMS proof mass attached to the piezoelectric AIN film. The wake-up ASIC is designed to consume very low energy. The wireless module is in a hibernate mode until being woken up by ASIC which then transmits the alert signal for a very short duration. Therefore, the system only consumes minimum quiescent energy, drawing significant power only when triggered.

As stated earlier, ARISE leverages Sandia's N-Zero vibratory sensor, is not simply a replacement for a conventional wired, high bandwidth accelerometer, but a paradigm shift to very low quiescent-power, narrow frequency band targeted acceleration-threshold sensor that operates with minimal power consumption but serves as a definitive trigger, characteristic of the system, for the activation of more extensive diagnostics and system level communication. For most aircraft operations, a "reliable alert" with low False Alarm Rate (FAR) that can lead to a later maintenance action is extremely valuable.

The description of the low power wireless module is not included in this paper. The goal is to validate the functionality of the sensor/ASIC package.

\section{ROTATING MACHINERY TESTING}

A rotating machine was selected to demonstrate the ARISE technology. The machine designated was known to experience gradual progressive bearing failure. To investigate the failure characteristics and establish the proper vibration-based detection method, a series of progressive damage tests were conducted. The test starts with a newly assembled machine, which were then subjected to accelerated operational loads/conditions, such as shaft unbalance, bearing overload, etc. The test ran continuously until the machine fails, and whereupon the machine was disassembled to verify the bearing failure mode. The machine was instrumented with conventional piezoelectric accelerometers with a sampling rate of $50 \mathrm{kHz}$.

The ARISE sensor is designed to target a specific known speed-independent resonant frequency. As stated, identification of this frequency peculiar to the selected machine must be identified from the rotating machinery prior to the design of the sensor. At this frequency the degradation evolution can be clearly observed, especially Stage 2 damage.

For test and demonstration purposes, one data set was selected which had damage progression in a relatively short duration and a clear failure mode. In this test, the bearing was intentionally overloaded leading to high vibration levels and accelerated damage progression. The machine failed in 69 minutes, and the tear down inspection confirmed a bearing failure. The overall waveform history from one accelerometer attached to the machine outer frame is shown in Figure 7, the zoom-in waveform is also shown. The machine ran at constant speed and the overload was applied starting at 19 minutes. After 65 minutes, the machine experienced excessive vibration up to $150 \mathrm{G}$ and was shut down at 69 minutes.

The vibration increased from $20 \mathrm{G}$ to $150 \mathrm{G}$ after 65 minutes, thus the machine had reached an unsustainable stage. Usually this is categorized as Stage 4 damage and the machine is close to the failure. For CBM purpose, the damage must be detected prior 65 minutes.
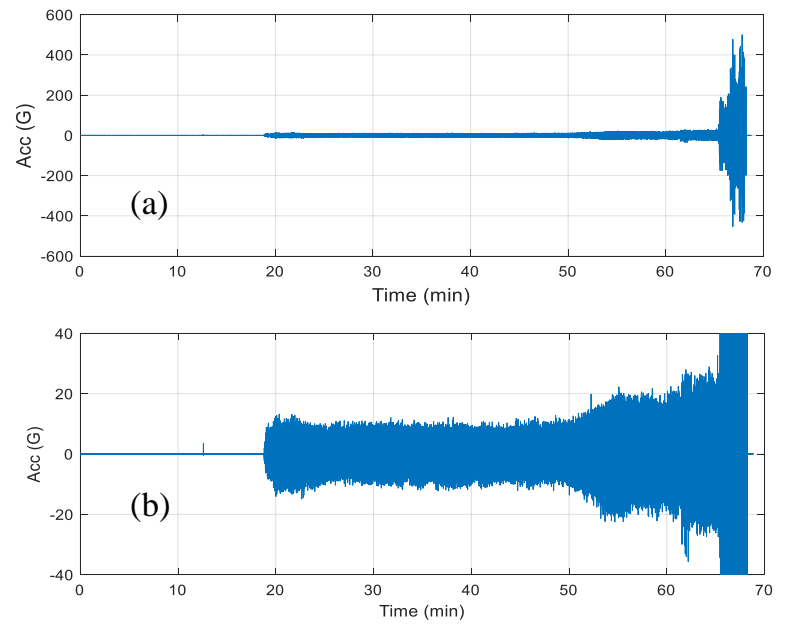

Figure 7. (a) Measured vibration waveform (b) zoomed waveform

To identify the representative natural frequency of the associated failure mode, the time-frequency analysis (Short Time Fourier's Transformation) is performed and the waterfall plot is shown in Figure 8, in which the acceleration is converted to displacement, since the output voltage of AIN-based piezoelectric sensor is proportional to the displacement. As seen in Figure 8, there are two, so called speed-independent, carrier frequency bands: around $1350 \mathrm{~Hz}$ and $3400 \mathrm{~Hz}$, which show most sensitivity to the damage progression. These two frequencies are the desired frequencies for ARISE. Later, $1350 \mathrm{~Hz}$ is selected for technology demonstration, because of its relatively higher signal level. Tests of other same pedigree machines show similar frequency bands with very minor variation. 


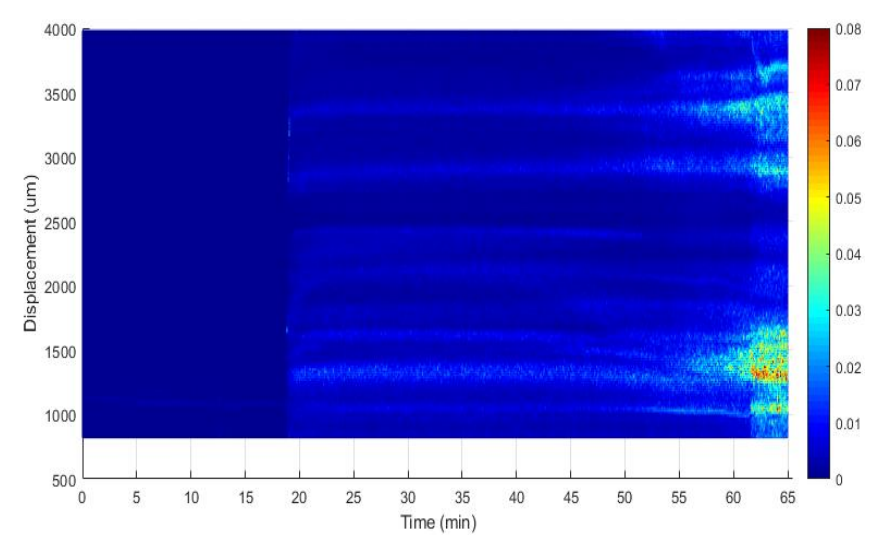

Figure 8. Time-Frequency waterfall plot

A bandpass filter at $1335 \mathrm{~Hz}$ is applied to the raw signal in order to evaluate the damage stage from the vibration magnitude at the resonance frequency, as shown in Figure 9 (a). The bandwidth of the filter is $+/-10 \mathrm{~Hz}$ to represent the resonator's narrow bandwidth. This raw vibration waveform is fed into a 1-DOF dynamic model of the resonator to simulate the output from the AIN film, shown in Figure 9(b), in which the resonance frequency is set to $1335 \mathrm{~Hz}$ with a $\mathrm{Q}$ factor of 140. The piezoelectric parameters of the sensor in the model are not calibrated through experiments, therefore the simulated sensor output isn't used directly to determine the threshold of ASIC. The general trend of the filtered accelerometer response and the simulated sensor output show consistent monotonically growing response. Based on the vibration amplitude, the general damage categories are assigned and shown in Table 2. Our objective is to detect at the earliest stage much prior to a catastrophic failure. Accordingly the ASIC threshold, i.e., g level at which the ASIC is triggered, is set to $0.2 \mathrm{G}$. The simulated sensor output in Figure 9(b) provides an estimate of threshold trigger voltage, which is experimentally determined in the following section.
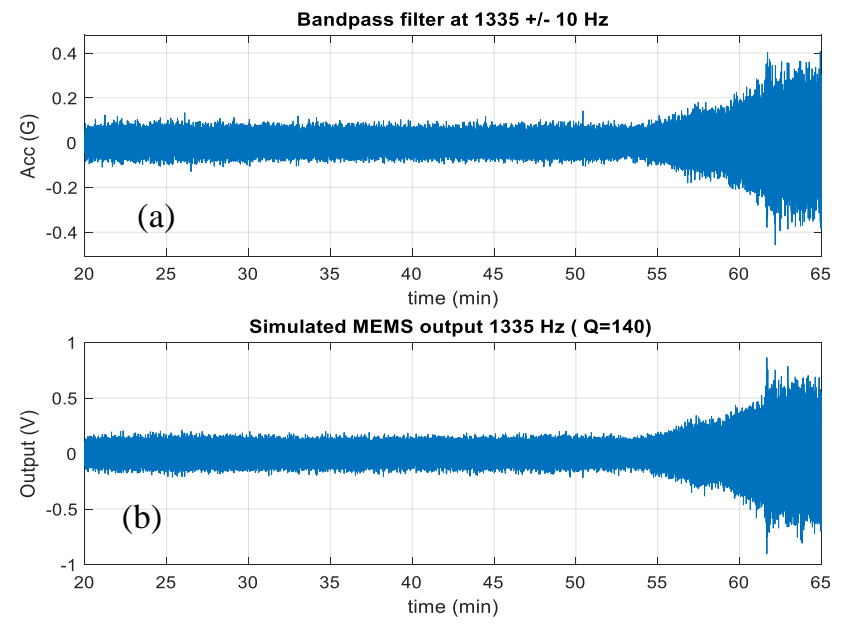

Figure 9. (a) Band-pass filtered vibration (b) Simulated sensor output using SNL 1DOF model

\begin{tabular}{|c|c|c|}
\hline $\begin{array}{c}\text { Time } \\
\text { Mark(min) }\end{array}$ & Condition & $\begin{array}{c}\text { Vibration } \\
\text { Level }\end{array}$ \\
\hline $18 \sim 54$ & Health & $0.1 \mathrm{G}$ \\
\hline $54 \sim 62$ & Initiation & $0.1 \sim 0.2 \mathrm{G}$ \\
\hline $62 \sim 65$ & Impending Failure & $0.2 \sim 0.5 \mathrm{G}$ \\
\hline$>65$ & Failure & $>0.6 \mathrm{G}$ \\
\hline
\end{tabular}

Table 2. Damage stage and vibration level

\section{Sensor Performance Test}

After the resonance frequency was identified and selected, the MEMS resonators were fabricated by SNL. The sensor was then packaged and tested with the shaker. A sine sweep test was conducted from $1200 \sim 1500 \mathrm{~Hz}$ at $4 \mathrm{~Hz} / \mathrm{sec}$ with three constant g levels: 0.2, 0.4, 0.6G. The sensor output was measured and the results are shown in Figure 10. Several sensors were tested and the one with a resonant frequency at $1335 \mathrm{~Hz}$ was down-selected. The $\mathrm{Q}$ factor (without vacuum) is computed to be 140. The measured sensor output in Figure 10 is lower than what the ASIC actually receives, this is because the impedance of Data Acquisition System is lower than the ASIC.

Once the MEMS sensor's resonator function was verified, it was packaged together with the ASIC. Then the packaged sensor was tested again with the same sine sweep profile at $0.2 \mathrm{G}$. The threshold trigger voltage of the ASIC was set to $87 \mathrm{mV}$, and as seen in Figure 11, the ASIC was triggered at the pre-set level when the sweep frequency coincided with the resonant frequency.

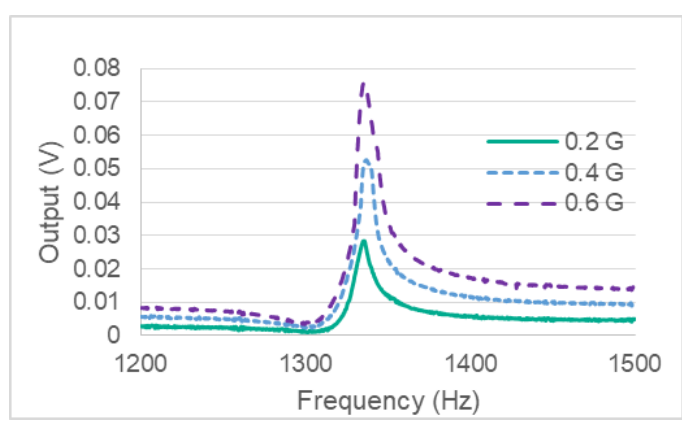

Figure 10. Sensor frequency response curve

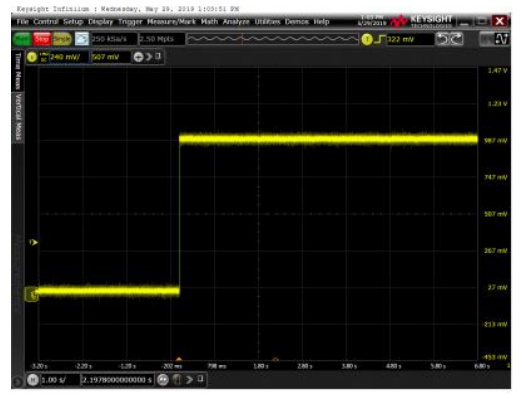

Figure 11. ASIC trigger test 


\section{VALIDATION TEST}

After the functionality check of the assembled sensor/ASIC package, it was tested with the more realistic vibratory input from the rotating machine shown earlier in Figure 7. The test setup is shown in Figure 12. The measured acceleration is fed into B\&K LASER controller, which has capability to control the shaker to replicate the waveform. Accelerations of the shaker table and the packaged sensor are monitored.

The actual test lasted over one hour. To accelerate the testing, a condensed testing profile was created from selected segments. As illustrated in Figure 13 (a), 10 seconds signals after every 4 minutes, marked by a circle, are selected and stitched together to form a shorter testing profile. The testing profile covers all major damage evolution stages. A $7 \mathrm{kHz}$ low-pass filter was applied to the input test data to accommodate the bandwidth of shaker. The final shaker input profile is shown in Figure 13(b).

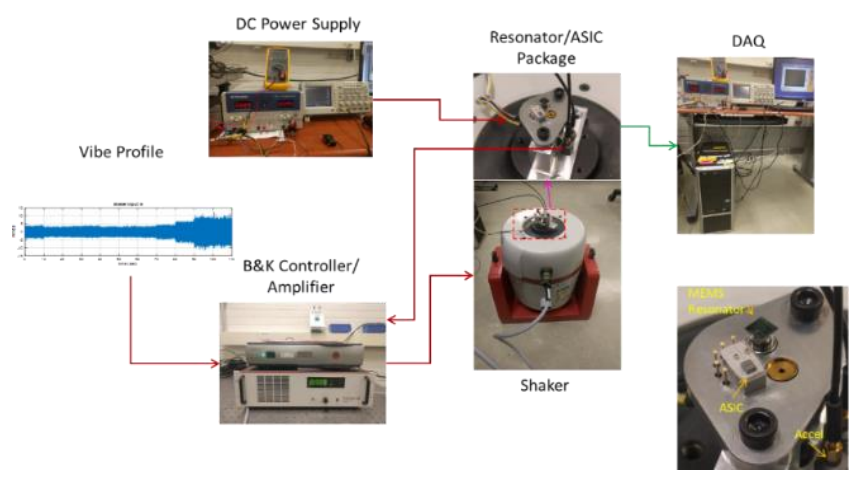

Figure 12. ARISE Validation Test setup
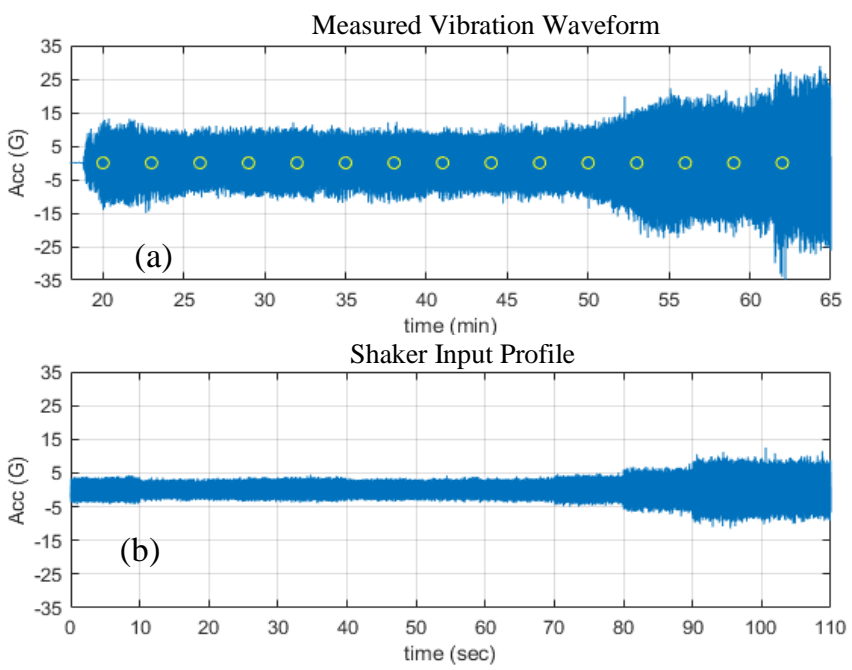

Figure 13. (a) Data segment selection (b) Reduced testing vibration profile

The measured shaker response under the given input profile is shown in Figure 14(a), which shows good agreement with the input profile. And the corresponding sensor output is simulated with 1DOF model and shown in Figure 14(b). As discussed in the previous section, the fault should be detected at $<65$ minutes in Figure 13(a), which is 100 110 second into the shaker input profile shown in Figure 14(a). The ASIC successfully tripped at 101 seconds shown in Figure 14(c). The test result showed good consistency from several repeats. The same test was repeated on other sensors with similar results confirming the capability of the low power sensor.
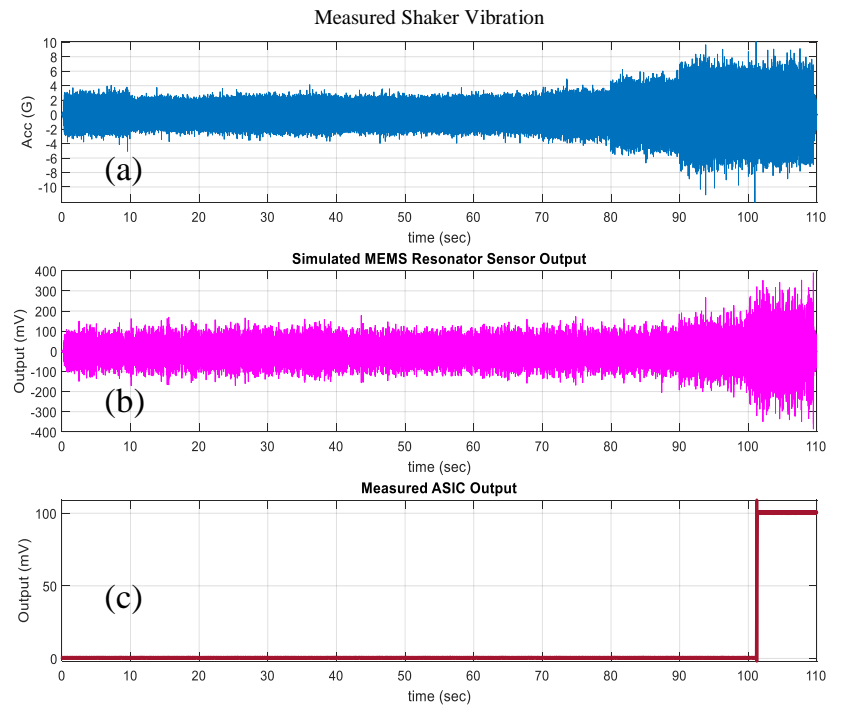

Figure 14. (a) Measured shaker vibration, (b) Simulated sensor output with 1DOF model, (c) Measured ASIC output

\section{CONCLUSIONS AND DISCUSSION}

The ARISE technology, which leverages N-Zero investment, has shown to be a viable means of low power wireless monitoring of rotating machines. An accelerated failure test which spans the various stages of progressive failure was selected, for the demonstration of the concept. The functionality of the sensor and sensor/ASIC package were checked separately, each of them meeting the design requirements.

\section{ACKNOWLEDGEMENT}

Special thanks to Mike Nesnidal, Dosi Dosev, Weibin Zhang of Collins Aerospace, and Robert Reger, Sean Yen of Sandia National Laboratories.

\section{DisClaim}

The views, opinions and/or findings expressed are those of the author and should not be interpreted as representing the official views or policies of the Department of Defense or the U.S. Government. 


\section{REFERENCES}

Lee, J., Wu, F., Ghaffari, M., Liao, L., Siegel, D., (2014). Prognostics and health management design for rotary machinery systems-Reviews, methodology and applications, Mechanical Systems and Signal Processing. vol. 42, pp. 314-334

Randall, R.B., Antoni, J., Chobsaard,S., (2001). The relationship between spectral correlation and envelope analysis in the diagnostics of bearing faults and other cyclostationary machine signal, Mechanical Systems and Signal Processing, September, vol. 15, issue 5,

Chen, Y., Chaudhry, Z., Dempsey, P., (2014). A Novel Gear Condition Monitoring Method based on Transient Impulse Response, Annual Forum Proceedings - AHS International, May 20-23, Montreal, Canada .

Griffin, B. A, McClanahan, R., Clews, P. J., Bryan, G. M., Barnard, C. A., Young, A. I., Henry, M. D., Young, T. R., Stevens, J. E., Summers, S. M., and Lemon, S., (2016). Resonant Aluminum Nitride Microphones as Passive Acoustic Filters, Internal SAND Report, January, SAND2016-0435.

Griffin, B. A, Nordquist, C. D., Eichenfield, M., Barney, B., Siddiqui, A., Grine, A., Henry, M. D., Reger, R. W., Satches, M., Young, A. I., Pluym, T., Delaney, M. A., Lepkowski, S. and Yen, S., (2017). A Near-Zero Power Sensor Suite Using Piezoelectric Microelectromechanical Systems, GOMACTech, March 22, Reno, NV.

Reger, R. W., Clews, P. J., Bryan, G. M., Keane, C. B., Henry, M. D., and Griffin, B. A., (2017). Aluminum Nitride Piezoelectric Microphones as Zero-Power Passive Acoustic Filters, IEEE Transducers, June 18-22, Kaoshuing, Taiwan.

Reger, R. W., Barney, B., Yen, S., Satches, M., Wiwi, M., Young, A. I., Delaney, M. A., and Griffin, B. A., (2017). Near-Zero Power Accelerometer Wakeup System, IEEE Sensors, Oct 29-Nov 1, Glasgow, Scotland.

\section{BIOGRAPHIES}

Dr. Yan Chen is a principle engineer at RTRC specializing in system dynamics and vibration, vibration control, modal testing, and bearing/gear PHM. Dr. Chen has extensive experience on the rotary machinery PHM, especially vibration-based bearing and gear fault detection.

Dr. Zaffir Chaudhry is a Research Fellow at RTRC specializing in mechanical systems design, vibrations, bearing tribology and diagnostics. Dr. Chaudhry has 25 years of engineering and $\mathrm{R} \& \mathrm{D}$ experience and has led many multidisciplinary projects to TRL6 and in to product release. He holds a PhD in Mechanical Engineering from Virginia Tech where we was deeply involved with the foundational work on health monitoring of machines and structures. Dr. Chaudhry has 44 patents and is author of over 70 publications on actuation, sensing, health monitoring and mechanical systems.

Dr. Joseph Mantese is a Research Fellow at RTRC specializing in electronic/optical materials, components, sensors, actuators, and packaging. Dr. Mantese was Department Head and Senior Fellow of Delphi Research Laboratories responsible for developing advanced technologies for automotive systems. He is a Fellow of the: Connecticut Academy of Science, American Physical Society, and the Materials Research Society. Dr. Mantese has 43 patents and is author of over 100 peer reviewed papers on electronic/optical materials, sensors, actuators, and MEMS. 